\title{
Malignant Hard Palate Neoplasm
}

National Cancer Institute

\section{Source}

National Cancer Institute. Malignant Hard Palate Neoplasm. NCI Thesaurus. Code C3528.

A primary or metastatic malignant neoplasm that affects the hard palate. 